\title{
HOW RADIO SOURCES STAY YOUNG
}

\author{
J.A. EILEK \\ New Mexico Tech \\ Socorro, NM, USA
}

\section{The Sources Are Older Than They Look}

In studying the dynamical evolution of radio galaxies, I find that the dynamical ages of the sources are older than the ages inferred from spectral breaks, typically by a factor $\sim 10$. This suggests to me that the spectral ages are misleading.

I model Type I sources as steady flows, described by energy and mass transport integrated across the tails. From this, data on surface brightness and tail width give me the mean velocity field as a function of distance down the tail, and thus the dynamical age of plasma along the tail. Assuming the magnetic field is initially in equipartion, and evolves by flux-freezing along the tail, also gives me the rate of spectral steepening predicted by the standard aging model. When I apply this (in Eilek 1996a) to the two-frequency data of O'Donoghue, Owen \& Eilek (1990), I find that the dynamical age at the end of the tail is $\sim 10-100$ times longer than the inferred spectral age. That is, the spectrum steepens much more slowly than it should.

I model Type II sources globally, in terms of their linear size and lobe volume. I assume again they are driven by steady jets, supplying energy and momentum to the extended sources. The source size is determined by momentum flux, and the source volume by pressure-driven expansion. Measurements of these two quantities tell us the age and beam power of the source. Assuming the magnetic field in the lobe is supplied by the jet, I can again predict their synchrotron age from standard theory. When I apply these arguments (in Eilek 1996b) to the data of Alexander \& Leahy (1986), I find they should be synchrotron old: their integrated spectra should be much steeper than observed. I also apply this analysis to modelling size and luminosity functions of nearby Type II sources, with the same conclusions: the spectra of these sources do not age as fast as they should. 


\section{Possible Reasons}

These contradictions are based on the standard model for synchrotron aging. This model assumes a uniform $B$ field, particles well mixed with this field, and that only radiative (or adiabatic) losses affect the particle spectrum after its initial injection. These are restrictive assumptions: the situation might well be more complex. In particular the magnetic field is very likely not to be uniform throughout the source.

One possibility is in situ acceleration. While several types of reacceleration are possible, most share a characteristic result: acceleration in the presence of synchrotron losses leads to a high-energy break in the electron distribution, at some energy $E_{c}$ where acceleration and loss rates balance. In diffuse radio sources, away from shocks, the acceleration is likely to be from stochastic turbulence. This can create an electron distribution peaked at $E_{c}$, rather than a power law (Borovsky \& Eilek 1986). Such a distribution can, however, produce a power-law spectrum if the particles sit in an inhomogeneous $B$ field with a power law volume distribution (Eilek \& Arendt 1996). The rate of evolution of $E_{c}$ depends on the local balance of turbulent or shock energy with the $B$ field. Thus, when we measure the spectral steepening we measure the turbulence level, not the age of the plasma.

Another possibility is that the $B$ field is inhomogeneous, and that the electrons move only slowly into high-field regions. Eilek, Melrose \& Walker (1996) describe this. They first follow the time evolution of electrons diffusing into a high-field region. They calculate the spectral steepening rate; it is slower than would be the case if the particles were initially well-mixed with the strong field. They also follow the evolution of particles moving between the high and low field regions in a leaky box model. With injection in the low-field region, they find that spectral steepening stops when leakage into the high-field region balances synchrotron losses. At this point, $E_{c}$ reaches a constant value. Using cross-field diffusion, they find that the diffusion rate in low turbulence levels predicts an $E_{c}$ which is consistent with observed spectral breaks in the $\sim \mathrm{GHz}$ range. Thus, when we measure the spectral steepening we measure the diffusion rate, not the age of the source.

\section{References}

Alexander, P, \& Leahy, J. P. (1986), MNRAS, 225, 1.

Borovsky, J. O. \& Eilek, J. A. (1986), ApJ, 308, 929.

Eilek, J. A. (1996a), submitted to $A p J$.

Eilek, J. A. (1996b), in preparation.

Eilek, J. A. \& Arendt, P. N. (1996), $A p J$, in press.

Eilek, J. A., Melrose, D. B. \& Walker, M. A. W. (1996), submitted to ApJ.

O'Donoghue, A. A., Owen, F. N. \& Eilek, J. A. (1990), ApJ Supp, $72,75$. 